\title{
On the probability of breakdown in participation games
}

\author{
Pim Heijnen
}

Received: 14 December 2005 / Accepted: 31 August 2008 / Published online: 23 September 2008 (C) The Author(s) 2008. This article is published with open access at Springerlink.com

\begin{abstract}
In this paper I analyze a participation game i.e. a public good game where contributions to the public good are binary (people either participate or not participate). Although variants of this game have been studied extensively, most previous work takes the benefit of provision of the public good to be independent of the number of players that contribute and show that the probability of breakdown, i.e. the probability that no one participates, is increasing in group size. Here this assumption is dropped. I show when the probability of breakdown is decreasing in group size and also present sufficient conditions under which the probability of breakdown is increasing in group size. Moreover I show that for large groups this probability is non-negligible in the limit and that the expected number of participants is less than one. Two economic examples, concerning R\&D and debt overhang, are discussed.
\end{abstract}

\section{Introduction}

Kitty Genovese was murdered in New York City in 1964 while 38 neighbors watched without calling the police. This story is consistent with evidence from both social psychology and game theory that suggests that people are less likely to help someone in need the more people surround them (Dixit and Skeath 1999, pp. 388-392;

\footnotetext{
The proof of Proposition 5 owes much to the useful comments and suggestions of Bert Schoonbeek. The author would like to thank Peter Kooreman, Linda Toolsema, Marco Haan, Allard van der Made, José-Luis Moraga-González, Florian Wagener, Maurice Koster, an anonymous referee and the associate editor for helpful comments and discussions. Financial support by the Netherlands Organization for Scientific Research (NWO) is gratefully acknowledged.

P. Heijnen $(\varangle)$

CeNDEF, Department of Quantitative Economics, University of Amsterdam,

Roetersstraat 11, 1018 WB Amsterdam, The Netherlands

e-mail: p.heijnen@uva.nl
} 
other references are Rasmusen 2001, pp. 77-79, Harrington 2001, and Osborne 2004, pp. 130-133). At least from the perspective of game theory, this seems puzzling.

Assume that seeking help is costly and that rescue is the preferred outcome. Then, although freeriding decreases the probability that each individual acts, it is by no means certain that the probability that the group as a whole fails to act (i.e. what I call the probability of breakdown), also increases.

Under the more restrictive assumption that the cost and benefit of rescuing are constant, equilibrium conditions ensure that the probability, that any of the other people fails to act, stays constant. A direct consequence is that the probability of breakdown is increasing in group size. In general however, this probability will only increase, if the freeriding effect is strong enough. The question then is, if it is possible for large groups to have a lower probability of breakdown and when this might occur.

I answer these questions for a more general class of problems, which I call participation games. A participation game is a binary public good game where the player either contributes to the public good (or participates/acts) or not. Moreover, just one player needs to contribute to the public good in order for everyone to enjoy the benefits. The cost and benefit of the provision of the public good may not be constant, but may depend on the number of players acting.

The contributions of this paper are the following. First, I characterize all Nash equilibria. While there are many Nash equilibria, the paper focuses on the symmetric mixed-strategy Nash equilibrium. Second, I show that there are public good games which have the property that the probability of breakdown decreases as the group becomes larger. Hence, large public good games can be more efficient than smaller games. In particular, sufficiently strong positive externalities seem to be required for this to occur. However, finally, I show that there are fairly mild conditions such that the probability of breakdown increases.

Anderson and Engers (2007) present a different generalization of this problem, but they only consider the case where the incentive to participate is either increasing or decreasing in the number of other players that participate. Contrary to their approach, I impose no monotonicity requirements.

The defining characteristic of participation games is that it is optimal to participate if and only if no one else participates. Below I will in short discuss the work on participation-like games, which points to its relevance in numerous applications.

Palfrey and Rosenthal (1983) offer one of the first systematic analyses of binary public good games. They consider the following variant: there are two groups of individuals. The group with the most individual contributions wins. An example would be the US presidential elections. Given the behavior of the other group, there is an incentive to free ride.

Haan and Kooreman (2003) show that in this game even if one group has an arbitrarily large potential majority (if everyone in their group will vote), it will not always win. This is due to the fact there is a subgame where members of the majority group have to decide whether they are going to contribute to the public good (i.e. voting to help their group win).

Mukhopadhyay (2003) extends the basic model with incomplete information. Mukhopadhyay examines the Condorcet jury theorem which states that a larger jury makes a more accurate verdict. Mukhopadhyay argues that if paying attention in court 
is costly (i.e. boring) and every juror wants to make an accurate verdict (for which they have to pay attention), then in a large jury there is an increasingly large probability that no one will pay attention and the Condorcet jury theorem will not hold. This conclusion is not altered when the jurors receive a signal, whether the defendant is likely to be guilty or not, prior to the court case.

Although variants of this game have been studied extensively, previous work takes the benefit of provision of the public good to be independent of the number of players that contribute. While the model presented here cannot be seen as a direct generalization of most of the papers mentioned in the previous paragraphs, at heart the results they all report are due to the presence of a participation (sub)game.

The paper is organized as follows: in Sect. 2 I introduce the participation game, derive all its equilibria and show that the probability of participating for each player is decreasing in group size. Section 3 discusses the probability of breakdown. I show when this probability is increasing and give sufficient conditions. Section 4 gives two economic examples concerning R\&D and debt overhang (based on Kaneko and Prokop 1993). Section 5 concludes.

\section{The participation game}

Denote the group of $n+1$ players by $N=\{1, \ldots, n+1\}$. Each player $i \in N$ can choose to participate $\left(a_{i}=1\right)$ or not participate $\left(a_{i}=0\right)$. For player $i$, define $A_{i}=\sum_{j \neq i} a_{j} \in\{0, \ldots, n\}$ as the total number of other players who participate. The utility of player $i$ depends only on $a_{i}$ and $A_{i}$. Denote the payoff function by $\pi\left(a_{i}, A_{i}\right)$. Players have identical (von Neumann-Morgenstern) preferences and move simultaneously.

Assumption 1 Participation is costly if at least one other player participates: for all $k=1, \ldots, n$ we have $\pi(1, k)<\pi(0, k)$.

Assumption 2 If nobody participates, the best-response is to participate: $\pi(0,0)<$ $\pi(1,0)$.

I refer to the game defined above as the participation game.

As an example of a participation game, consider the murder of Kitty Genovese as discussed in the introduction. Here a group of $n+1$ people observe someone attacking Kitty. Each person has the opportunity to call the policy. Each person receives a payoff of $b$ if Kitty is saved, but a person who actually actually calls the policy incurs a cost $c$. The payoff function for each player $i$ is:

$$
\begin{aligned}
& \pi(0,0)=0, \\
& \pi(0, k)=b, \quad \text { for all } k>0, \\
& \pi(1, k)=b-c, \quad \text { for all } k \geq 0,
\end{aligned}
$$

where $b, c>0$ and $b>c$. I will refer to this game as the participation game with constant cost and benefits. 
Proceeding with the participation game, it is easy to see that there is no symmetric equilibrium in pure strategies (cf. Lemma 7 in Appendix A). To see this, suppose that everyone else participates. Then by Assumption 1 it is optimal to not participate. However, when no one else participates, by Assumption 2 it is optimal to participate. Hence neither of the pure strategies is a best response against itself.

However, there is a symmetric mixed equilibrium that can be derived as follows. Suppose that $n$ people participate with probability $p$. The $(n+1)$ th person should be indifferent between participating and not participating. Hence in equilibrium:

$$
\sum_{k=0}^{n}\left(\begin{array}{l}
n \\
k
\end{array}\right) p^{k}(1-p)^{n-k} \theta_{k}=0,
$$

where $\theta_{k} \equiv \pi(0, k)-\pi(1, k)$. This parameter signifies the incentive to not participate if $k$ people are already participating. From Assumptions 1 and 2, it follows that $\theta_{0}<0$ and $\theta_{k}>0$ (for $k>0$ ). In the symmetric Nash equilibrium everyone participates with probability equal to a root of (1) in the unit interval.

Define the function

$$
f_{m}(p)=\sum_{k=0}^{m}\left(\begin{array}{l}
m \\
k
\end{array}\right) p^{k}(1-p)^{m-k} \theta_{k}
$$

where $0<m \leq n$. Let $p_{m}$ denote a root of $f_{m}(\cdot)$. It turns out there there are no equilibria where players who mix between actions choose different mixing probabilities (cf. Lemma 12 in Appendix A). Therefore all equilibrium mixing probabilities are determined by roots on the unit interval of the function $f_{m}(p)$. These roots correspond to an equilibrium where $m$ players mix between participating and not participating and the remaining $n+1-m$ do not participate (which is what Lemmas 9-11 in Appendix A demonstrate).

Before we continue the examination of the properties of the symmetric equilibrium, I first present the following proposition that characterizes all equilibria.

Proposition 3 (Characterization of equilibria). There are exactly $2^{n+1}-1$ equilibria. In each of these equilibria, each member of a set $M \subseteq N$ (with the exception of the empty set) participates with probability $p>0$ and each member of $N \backslash M$ does not participate. Moreover:

(i) If $\# M=1$, then $p=1$ (equilibrium in pure strategies).

(ii) If $\# M>1$, then $p$ is the unique root of $f_{\# M-1}(\cdot), 0<p<1$.

Outline of the proof. First, it is shown that there are no symmetric pure strategy equilibria (Lemma 7). Second, for any player, there is a unique asymmetric equilibrium in pure strategies, where one player participates with probability 1 and other players do not participate (Lemma 8). Third, for any non-empty subset of players with cardinality greater than one, there is a unique symmetric equilibrium in mixed strategies, where all players in the subset participate with probability $p \in(0,1)$ and players not in the set do not participate (Lemmas 9, 10 and 11). Since there are no asymmetric 
strictly mixed equilibria (Lemma 12), all possible equilibria are the ones given by Lemmas 8-11. See Appendix A for the proof.

From the Proposition we see that there is a unique symmetric mixed equilibrium, where everybody participates with probability $p_{n}$, the unique root of the function $f_{n}(\cdot)$. From now on my focus will be on the symmetric equilibrium. This equilibrium has the following property:

Proposition 4 (Effect of group size on individual participation probability). In the symmetric equilibrium of the participation game we have $p_{n+1}<p_{n}$ : the probability of participation declines if group size increases.

Outline of the proof. It is shown that $f_{n+1}\left(p_{n}\right)>0$ and $f_{n+1}(0)<0$. Since $p_{n+1}$ is the unique root of $f_{n+1}(\cdot)$, it must be in the interval $\left(0, p_{n}\right)$. See Appendix B for the proof.

This proposition shows the freeriding problem that is inherent to public good games.

\section{The probability of breakdown}

The probability that no one participates is given by $S_{n} \equiv\left(1-p_{n}\right)^{n+1}$. Call $S_{n}$ the probability of breakdown. I am interested in how $S_{n}$ depends on $n$.

Returning to the participation game with constant cost and benefits, where the benefit to each player if the public good is provided is $b$ and the cost of provision of the public good is $c$ (both benefit and cost do not depend on how many people participate), it has been shown that the probability of breakdown is always increasing.

To find the Nash equilibrium observe that in the equilibrium the expected payoff of participating, $b-c$, is equal to the expected payoff of not participating, $b[1-(1-$ $\left.\left.p_{n}\right)^{n}\right]$ ), where the latter expression is the probability that at least one other person participates. Equating these two expressions, after some manipulation, yields

$$
\left(1-p_{n}\right)^{n}=1-\frac{c}{b} \text {. }
$$

Now we see that $S_{n}=\left(1-\frac{c}{b}\right)\left(1-p_{n}\right)$. Since $p_{n}$ is decreasing in $n$, it follows that $S_{n}$ is increasing. This is a property that seems to hold for most participation games.

The following two propositions present sufficient conditions under which the probability of breakdown increases. The first condition, presented in Proposition 5, is weak in the sense that it only depends on the ratio of $\theta_{0}$ and $\theta_{1}$. The second condition, presented in Proposition 6, states that monotonicity of the payoffs, i.e. $\theta_{1}<\theta_{2}<\ldots$, is also a sufficient condition.

Proposition 5 (Weak sufficient condition for the probability of breakdown to increase). If $\left|\theta_{0}\right|<\left|\theta_{1}\right|$, then

(i) $S_{n+1}>S_{n}$ for all $n \in \mathbb{N}$, and, moreover,

(ii) $S_{\infty}=\lim _{n \rightarrow \infty} S_{n} \geq e^{\frac{\theta_{0}}{\theta_{1}}}$,

(iii) the expected number of participants is less than one, i.e. $p_{n}(n+1)<1$. 
Outline of the proof. These results follows from the fact that not only is $p_{n+1}<p_{n}$, the difference between $p_{n+1}$ and $p_{n}$ cannot be arbitrarily small for given $n$ (Lemma 13). Using an upper bound for $p_{n+1}$ (given $p_{n}$ ), a lower bound for $S_{n+1}$ (given $S_{n}$ and conditional on $\left|\theta_{0}\right|<\left|\theta_{1}\right|$ ) is obtained (Lemma 14). Subsequently the lower bound is used to proof the results. See Appendix $C$ for the proof.

It is interesting to notice that if $\left|\theta_{0}\right|<\left|\theta_{1}\right|$, then the values of $\theta_{2}, \ldots, \theta_{n}$ are completely irrelevant: the probability of breakdown will increase regardless of these values.

The intuition behind this is the following. If the number of players increases, then the probability to participate in general will be small, i.e. from Proposition 5(iii) we know that $p_{n}<1 /(n+1)$. Hence for the individual decision maker, it is likely that at most one other player participates. Unless the incentive to participate if more than one other player participates is high, the player can ignore the probability that such indeed occurs.

If the unlikely event that more than one other player participates occurs, then the incentive to participate should be high. The scope for this is limited since high incentives means a low value of $\theta_{2}$. However, $\theta_{2}$ is positive by assumption. If $\left|\theta_{1}\right|>\left|\theta_{0}\right|$, then the incentives cannot be raised high enough to make $S_{n}$ go down.

Proposition 5 shows that, under a fairly weak condition only dependent on the ratio of $\theta_{0}$ and $\theta_{1}$, the probability of breakdown in binary public good games is increasing in group size. Hence, the result, that the probability of breakdown is increasing in group size, appears very robust to different payoff specifications.

Moreover, $S_{\infty}$ is always bigger than $\mathrm{e}^{-1} \approx 0.3679$. So, in large participation games satisfying the condition $\left|\theta_{0}\right|<\left|\theta_{1}\right|$, the probability of breakdown will be substantial.

Finally the Proposition shows that on average less than one person participates and thus the public good is underprovided (more than one being socially optimal). ${ }^{1}$

As an example of how to use Proposition 5 consider the following extension of the participation game with constant cost and benefits. Suppose that being recognized as a rescuer has merits of its own. If there are $k$ rescuers, then each obtains a hero bonus that translates into an extra payoff of $H / k$.

Now, $\theta_{0}=-[b+H-c]$ and $\theta_{1}=c-H / 2$. The game is a participation game if $H<2 c$ and $b+H-c>0$. The condition $\left|\theta_{0}\right|<\left|\theta_{1}\right|$ corresponds to $H<\frac{4}{3} c-\frac{2}{3} b$. So, if the hero bonus is sufficiently small, then the probability that no one rescues the drowner is still increasing in group size. Notice that for $n>2$ the equilibrium strategy will in general not be solvable without resorting to numerical methods.

For the second sufficient condition I will focus on monotonically increasing payoff functions. This corresponds to negative externalities. The purpose of this is twofold. First, it will yield another sufficient condition for the probability of breakdown to be increasing.

Second, the sufficient condition of Proposition 5 does not always yield "nice" conditions (as the application in Sect. 4.2 shows) whereas the condition for a negative externality is easier to interpret.

\footnotetext{
${ }^{1}$ The socially optimal outcome maximizes $\pi(0, k)(n+1-k)+\pi(1, k-1) k$ with respect to $k$.
} 
Suppose we have a game with $n+1$ players such that $\theta_{0}=-1$ (just a normalization) and, for all $k=1, \ldots, n, \theta_{k+1}>\theta_{k}$. If the $\theta_{k}$ 's are increasing, then $\pi(0, k)-\pi(1, k)$ is increasing in $k$. As more players participate the incentive to not participate becomes stronger; this is the case of a negative externality (cf. Anderson and Engers (2007) who focus solely on such monotonic payoff functions).

Construct an auxiliary game with parameters $\theta_{0}^{C}=-1$ and for all $k>0, \theta_{k+1}^{C}=\theta_{k}^{C}$ such that this game with $n+1$ players has the same symmetric equilibrium as the game above with increasing $\theta_{k}$ 's. Note that for every $n$ such an auxiliary game can be uniquely constructed. Observe that for the auxiliary game it is known that the probability of breakdown is increasing in $n$ (since this is the participation game with constant cost and benefits, which was analyzed at the beginning of Sect. 3).

Denote the equilibrium of the auxiliary game by $p_{n}^{C}$. By construction $p_{n}^{C}=p_{n}$, where $p_{n}$ is the probability of participation in the game with a negative externality. If we can show that $p_{n+1}<p_{n+1}^{C}$, then $\left(1-p_{n+1}\right)^{n+2}>\left(1-p_{n+1}^{C}\right)^{n+2}>\left(1-p_{n}^{C}\right)^{n+1}=$ $\left(1-p_{n}\right)^{n+1}$. From which we can conclude that the probability of breakdown in a game where, for all $k>0, \theta_{k+1}>\theta_{k}$, is increasing in group size.

I will now show that $p_{n+1}<p_{n+1}^{C}$. Examining the function $f_{n}(p)$, I observe that it is a weighted average of $\theta_{0}, \ldots, \theta_{k}$. The most weight is given to $k=\lfloor(n+1) p\rfloor$ since in a binomial distribution this is the most likely outcome. ${ }^{2}$ An equilibrium is then a $p$ which shifts the weights in such a way that the weighted average is zero.

In the auxiliary game, there exists an integer $m \in\{1, \ldots, n\}$ such that for $0<k<m$ we have $\theta_{k}^{C}>\theta_{k}$ and for $k \geq m$ we have $\theta_{k}^{C} \leq \theta_{k}$. This implies that $\theta_{n+1}^{C}<\theta_{n+1}$. So, in determining the equilibrium in the original game $p_{n+1}$ has to be smaller than $p_{n+1}^{C}$ to compensate.

The following proposition is thus obtained.

Proposition 6 (Strong sufficient condition for the probability of breakdown to increase). If, for all $k>0, \theta_{k+1}>\theta_{k}$, then $S_{n}$ is increasing in $n$.

Proposition 6 shows that if the parameters $\theta_{k}$ are increasing, then this is also a sufficient condition for the probability of breakdown to be increasing.

Note that in general if $S_{n+1}>S_{n}$, then:

$$
\left(1-p_{n+1}\right)^{n+2}>\left(1-p_{n}\right)^{n+1} \Leftrightarrow p_{n+1}<1-\left(1-p_{n}\right)^{\frac{n+1}{n+2}} \approx p_{n},
$$

where the approximation holds if $n$ large. This can be seen as an indication that it might be difficult to find examples where $S_{n+1} \leq S_{n}$ for $n$ large. However, one can construct examples in which $S_{n}$ is decreasing. I will now give such an example.

The counterexample. Consider the following game: $\theta_{0}=-1$, and $\theta_{k}=\alpha \beta^{k}$ for all $k \geq 1$. Assume that $\alpha>0$ and $0<\beta<1$. It can be shown that (see Appendix D):

$$
S_{n}=\left[\frac{\beta}{\sqrt[n]{(1+\alpha) / \alpha}-(1-\beta)}\right]^{n+1} .
$$

\footnotetext{
2 The truncation function is denoted by $L \cdot\rfloor$.
} 
Take $\beta=\frac{1}{25}$ and $\alpha=\frac{25}{4}$. Numerical calculations show that $S_{1}=0.0400, S_{2}=$ $0.0399, S_{3}=0.0378, S_{4}=0.0359$. So, $S_{4}<S_{3}<S_{2}<S_{1}$. In this example, $\theta_{2}, \theta_{3}$ etcetera are very close to zero and, hence, $\pi(0, k) \approx \pi(1, k)$ for $k \geq 2$ : that is, players are almost indifferent between participating and not participating. In this, what I will call the counterexample, there are strong positive externalities: the incentive to not participate, although present, becomes very small as $n$ increases. The counterexample also shows that large groups can exhibit a larger degree of cooperation than small groups.

As the counterexample shows, if the parameters $\theta_{k}$ decrease sufficiently fast, then the probability of breakdown can decrease. Hence, sufficiently strong positive externalities in the payoff (and $\left|\theta_{0}\right|>\left|\theta_{1}\right|$ ) might mitigate the paradox.

\section{Applications}

\subsection{R\&D and teamwork}

An economic application is teamwork. Suppose that the R\&D-department of a company consists of $n+1$ employees. Each employee has to decide whether to exert effort or not. Effort is interpreted as contributing to the research project.

If an employee exerts effort, then some innovation is created and the employee bears a cost $c$. The employee values this innovation, but the value only depends on the number of employees that have exerted effort (including the employee himself). Denote this value by $V(k)$, where $k$ is the number of participants, and assume, without loss of generality, that $V(0)=0$.

The payoff function, using the notation of Sect. 2, is $\pi\left(a_{i}, A_{i}\right)=V\left(a_{i}+A_{i}\right)-a_{i} c$. Note that

$$
\theta_{k}=V(k)-V(k+1)+c .
$$

To fit into my framework, the following restrictions are needed:

$$
\begin{aligned}
& \theta_{0}=-V(1)+c<0 \\
& \theta_{k}=V(k)-V(k+1)+c>0 \text { for all } k \geq 1
\end{aligned}
$$

If we assume that $V(\cdot)$ is concave, then the $\theta_{k}$ 's are increasing in $k$, i.e.

$$
\frac{\partial \theta_{k}}{\partial k}=V^{\prime}(k)-V^{\prime}(k+1)>0
$$

Under concavity of $V(\cdot)$, the probability that the innovation is not discovered is increasing in the number of coworkers. Concavity can be interpreted in the following manner: the effort of each employee increases the value of the innovation, but the additional value diminishes. 
This lack of "synergy" does not imply that team work is necessarily unwanted. In a cooperative equilibrium, that maximizes $n V(k)-c k$ with respect to $k$, the optimal number of employees that exert effort can be larger than one.

However, if a company puts together a research team, then some additional value of working together is expected. One could imagine that $V(\cdot)$ is S-shaped and teamwork would have increasing additional value if the number of contributors is below a certain number. ${ }^{3}$ In a natural manner, this yields non-monotonic $\theta_{k}$ 's and the result from Proposition 5 is useful.

The condition $\left|\theta_{0}\right|<\left|\theta_{1}\right|$ yields (from Proposition 5):

$$
V(2)<2 c \text {. }
$$

A possible interpretation of (10) is the following: if you could put in twice the effort, then this is not profitable. While you may benefit if others exert effort, it does not pay to do the extra effort yourself. This ensures that the probability that the innovation is not discovered is increasing in the number of coworkers. This example also shows that in not every application the assumption of monotonicity is innocuous.

\subsection{International debt overhang}

Another application, due to Kaneko and Prokop (1993), is debt overhang. Kaneko and Prokop (1993, p. 2) define it as follows:

The term "debt overhang" expresses a situation where a sovereign country has borrowed money from foreign banks and has not succeeded in fulfilling the scheduled repayments for some period. The existence of the debt overhang is a serious problem for the debtor country, which keeps the country in a bad economic situation.

I discuss a symmetric version of their model. In this game there are $n+1$ creditors and one country that is in debt. The debtor country is treated as part of the environment and not as an active player.

Each creditor holds a debt of size $D$. I assume that total outstanding debt $\bar{D}$ is constant, i.e. $(n+1) D=\bar{D}$. If the number of creditors increases, then they also hold a smaller share of total debt. This assumption is needed to ensure that below the parameters $\theta_{k}$ do not depend on $n$.

The creditors can sell off their debt at a secondary market price. The market price is given by the pricing function $P: \mathbb{R}^{+} \rightarrow \mathbb{R}^{+}$whose domain is total outstanding debt. For instance, if a creditor has an outstanding debt of $D$ and total outstanding debt is $\bar{D}$, then it can sell of this debt at $D P(\bar{D}) .{ }^{4}$ The larger the debt (and the higher the probability of default), the lower the price of buying back the debt.

\footnotetext{
${ }^{3}$ A function $V(k)$ is S-shaped if there exists $\bar{k} \in \mathbb{R}$ such that it is convex for all $k<\bar{k}$ and concave for all $k>\bar{k}$.

${ }^{4}$ This price is often far below 1; in the case of Bolivia in 1985 the price of buying back one dollar of debt was 5 cent (cf. Kaneko and Prokop 1993, p. 2).
} 
I follow Kaneko and Prokop (1993) and suppose that each creditor can either sell its debt now at a price $P(\bar{D})$ or wait for one period and then sell it. If the creditor postpones, then its debt will rise to $\beta D$, where $\beta>1$ is one plus the interest rate. However, the present value of the debt will stay $D$.

The price of buying back debt in the next period is $P(\beta(n+1-k) D)$, where $k$ is the number of other creditors that have not postponed. Each creditor has two actions: sell or postpone.

For the moment, and contrary to Kaneko and Prokop (1993), assume that $P(\cdot)$ is a decreasing and differentiable function. Since $P(\cdot)$ is a decreasing function, we have that:

$$
D P(\bar{D})>D P(\beta \bar{D}),
$$

where the present value of debt stays constant at $D$, but the price of buying back debt does change.

From (11) it follows that it is optimal for the creditors to sell if none of the other creditors have sold. I will show that this game is a participation game. In terms of the participation game selling is equal to participating and (11) is equal to Assumption 2. In order to qualify as a participation game, it must also be optimal to postpone if one other creditor sells:

$$
D P(\beta(\bar{D}-D))>D P(\bar{D})
$$

which boils down to $\beta<\frac{n+1}{n}$. This is equal to Assumption 1 if either the number of creditors is small or the interest rate is not too large. From Proposition 3, it follows that there is a unique symmetric equilibrium in this game.

Since the present value of debt, $D$, is constant for a creditor, the creditor is just interested in the price at which the debt is sold off. Hence, the payoff function is simplified to this price. The parameters of the model are defined as follows:

$$
\theta_{k}=P(\beta(\bar{D}-k D))-P(\bar{D}) .
$$

If $\beta<\frac{n+1}{n}$ and $P(\cdot)$ is decreasing, then $\theta_{0}<0$ and $\theta_{k}>0$ for all $k \geq 1$ ensuring that we have a participation game. Notice that:

$$
\frac{\partial \theta_{k}}{\partial k}=-\beta D P^{\prime}(\beta(\bar{D}-k D))>0,
$$

and thus $\theta_{k}$ is increasing in $k$. Hence, it follows directly from Proposition 6 , that an increase in the number of creditors implies that the probability that no one sells is increasing. Therefore having more creditors has a detrimental effect on debt overhang. Unlike the results of Kaneko and Prokop (1993), this result is valid for a small number of creditors.

Kaneko and Prokop (1993) do not assume that $P(\cdot)$ is decreasing: they merely assume that $P(0) \geq P(\bar{D})>P(\beta \bar{D})$. In this case, Proposition 5 can still be used to formulate sufficient conditions for the probability that no one sells to increase. First, to have $\theta_{k}>0$, for all $k \geq 1$, we need: 


$$
P(\beta(\bar{D}-k D))>P(\bar{D}) .
$$

The condition $\left|\theta_{0}\right|<\left|\theta_{1}\right|$ (from Proposition 5) yields

$$
2 P(\bar{D})<P(\beta \bar{D})-P(\beta(\bar{D}-D))
$$

If (15) and (16) both hold, then the probability that no one sells increases in the number of creditors. Remark that the sufficient condition of Proposition 5 does not yield easily interpretable conditions. The reasonable assumption of a decreasing pricing function, however, implies negative externalities and gives the desired result immediately.

\section{Conclusion}

This paper has investigated if it is possible in a participation game for large groups to have a lower probability probability of breakdown. The counterexample showed that this is possible, but it is not easy to find these example as strong positive externalities seem to be required for this phenomenon to occur. However, I presented two sufficient conditions under which the probability of breakdown turned out to be increasing.

Previous work by Anderson and Engers (2007) already showed how negative externalities will lead to a higher probability of breakdown, but I presented another condition only dependent on the incentive to participate if no or one other player participates. In the example about R\&D in Sect. 4.1, I argued that assuming negative externalities is not always as innocuous as it seems.

This paper only gives sufficient conditions. Clearly, necessary conditions or a full characterization of the set of parameters, for which the probability of breakdown is decreasing, are the next step.

Open Access This article is distributed under the terms of the Creative Commons Attribution Noncommercial License which permits any noncommercial use, distribution, and reproduction in any medium, provided the original author(s) and source are credited.

\section{Appendix}

\section{A Proof of Proposition 3}

It is convenient to present the following Lemmas first.

Lemma 7 There exists no symmetric pure strategy equilibrium.

Proof Suppose all players participate: $a_{i}=1$ and $A_{i}=n$ for all $i$. Each player then receives a payoff $\pi(1, n)$. However, by Assumption 1 the best-response is to not participate since $\pi(0, n)>\pi(1, n)$. Suppose all players do not participate: $a_{i}=0$ and $A_{i}=0$ for all $i$. However, by Assumption 2 the best-response is to participate.

Lemma 8 There are $(n+1)$ non-symmetric pure strategy equilibria. 
Proof Let $A_{i} \geq 1$ be the number of other people who participate. Those who participate have a payoff $\pi\left(1, A_{i}\right)<\pi\left(0, A_{i}\right)$ (Assumption 1). Clearly, if $A_{i} \geq 1$, this cannot be an equilibrium. This does not hold for $A_{i}=0$, since $\pi(1,0)>\pi(0,0)$ (Assumption 2). Only one player participates is an equilibrium. There are $(n+1)$ of these equilibria.

Lemma 9 There exists a unique symmetric mixed-strategy equilibrium where everyone participates with probability $p \in(0,1)$.

Proof We know that $p$ must be a real positive root of $f_{n}(\cdot)$ defined in (2). First, it has to be shown that it exists. Second, it has to be shown that it is unique.

It is straightforward to show that there is at least one root on the unit interval. Note that by Assumptions 1 and 2:

$$
\begin{aligned}
& f_{n}(0)=\pi(0,0)-\pi(1,0)=\theta_{0}<0 \\
& f_{n}(1)=\pi(0, n)-\pi(1, n)=\theta_{n}>0
\end{aligned}
$$

and remark that $f_{n}(\cdot)$ is a continuous function. This also implies that generically the number of roots is odd.

To proof unicity, notice that, for $p \neq 1, f_{n}(\cdot)$ can be rewritten as:

$$
f_{n}(p)=(1-p)^{n} \sum_{k=0}^{n}\left(\begin{array}{l}
n \\
k
\end{array}\right) r^{k} \theta_{k},
$$

where $r \equiv p /(1-p)$. This allows us to look at the roots of $\sum_{k=0}^{n}\left(\begin{array}{l}n \\ k\end{array}\right) r^{k} \theta_{k}$. Using Descartes' sign rule (see Atkinson 1989, p. 95), we now easily obtain that the root is unique.

In determining all asymmetric equilibria, it is useful to think of a person joining the group. Suppose that a group of $n+1$ players has already decided to participate with the equilibrium probability of $p_{n}$. If an extra person would join this group, what would this person do? The next Lemma shows that this person becomes a bystander: someone who does not participate.

Lemma 10 (The bystander). The following is an equilibrium: in a group of size $n+$ 2 , let $n+1$ players participate with probability $p_{n}$ and the remaining player (the bystander) will not participate.

Proof For the players who participate this is obviously an equilibrium strategy. For the bystander, the benefit of not participating over participating is given by:

$$
\begin{aligned}
\sum_{k=0}^{n+1}\left(\begin{array}{c}
n+1 \\
k
\end{array}\right) p_{n}^{k}\left(1-p_{n}\right)^{n-k} \theta_{k} & =p_{n}^{n+1} \theta_{n+1}+\sum_{k=0}^{n}\left(\begin{array}{l}
n \\
k
\end{array}\right) p_{n}^{k}\left(1-p_{n}\right)^{n-k} \theta_{k} \\
& \times\left[\frac{(n+1)\left(1-p_{n}\right)}{n+1-k}\right] .
\end{aligned}
$$


Note that (i) the first term on the RHS is positive and (ii) the second term on the RHS is also positive. In order to see the latter recall that $\sum_{k=0}^{n}\left(\begin{array}{l}n \\ k\end{array}\right) p_{n}^{k}\left(1-p_{n}\right)^{n-k} \theta_{k}=0$. Observe that this sum consists of one negative term at $k=0$ followed by exclusively positive terms. The new weighing factor $(n+1) /(n+1-k)$ puts more emphasis on these positive terms since it increases as $k$ increases. Hence, the bystander will not participate.

Lemma 11 Any non-empty subset of the group of players can play the symmetric mixed equilibrium for a subset of that size with the rest of the group being bystanders.

Proof Follows from Lemma 10.

Lemma 12 There exists no non-symmetric strictly mixed equilibrium.

Proof Suppose that each member of the group in an arbitrary mixed equilibrium participates with probability $p_{i}$, where $i=1, \ldots, n+1$. Suppose the focus is on a subset of the group without members $n_{1}, \ldots, n_{m}$. Call this subset $M \equiv N \backslash\left\{n_{1}, \ldots, n_{m}\right\}$. The probability $\operatorname{Pr}\left(k \mid n_{1}, \ldots, n_{m}\right)$ that $k$ players in this subset participate equals

$$
\sum_{K \subseteq M, \# K=k}\left\{\left(\prod_{j \in K} p_{j}\right)\left(\prod_{\ell \notin K}\left(1-p_{\ell}\right)\right)\right\} .
$$

This expression is obtained by summing over all subsets of $M$ of size $k$ and calculating the probability that for each of these subsets the $k$ people in this subset participate. If $k<0$ or $k>n-m$, then $\operatorname{Pr}\left(k \mid n_{1}, \ldots, n_{m}\right)$ is defined to be zero. Notice that $\operatorname{Pr}(k \mid i)=\operatorname{Pr}(k \mid i, j) \times\left(1-p_{j}\right)+\operatorname{Pr}(k-1 \mid i, j) \times p_{j}$.

For any $i, j \in N$, in a strictly mixed equilibrium the following should hold:

$$
\begin{aligned}
& \sum_{k=0}^{n} \operatorname{Pr}(k \mid i) \theta_{k}=0 \\
& \sum_{k=0}^{n} \operatorname{Pr}(k \mid j) \theta_{k}=0 .
\end{aligned}
$$

Both player $i$ and $j$ should be indifferent between participating and not participating. This is equivalent with:

$$
\begin{aligned}
& \sum_{k=0}^{n}\left[\operatorname{Pr}(k \mid i, j) \times\left(1-p_{j}\right)+\operatorname{Pr}(k-1 \mid i, j) \times p_{j}\right] \theta_{k}=0 \\
& \sum_{k=0}^{n}\left[\operatorname{Pr}(k \mid i, j) \times\left(1-p_{i}\right)+\operatorname{Pr}(k-1 \mid i, j) \times p_{i}\right] \theta_{k}=0,
\end{aligned}
$$

which implies that in equilibrium:

$$
p_{i}=p_{j}=\frac{\sum \operatorname{Pr}(k \mid i, j) \theta_{k}}{\sum \operatorname{Pr}(k \mid i, j) \theta_{k}-\sum \operatorname{Pr}(k-1 \mid i, j) \theta_{k}} .
$$


Therefore $p_{i}=p_{j}$ for any $i$ and any $j$. Hence there are no non-symmetric strictly mixed equilibria.

Proposition 3 follows easily from Lemmas 7 to 12 . Observe that for each subset of $N$ (with the exception of the empty set), there is an equilibrium where the members of the subset participate with the same probability and the other players do nothing. The number of non-empty subsets is $2^{n+1}-1$. Moreover, these probabilities are the unique root of $f_{m}(\cdot)$, where $m$ the number of players that participate. So there are exactly $2^{n+1}-1$ equilibria.

\section{B Proof of Proposition 4}

Note that $f_{n+1}(p)$ can be rewritten as:

$$
f_{n+1}(p)=p^{n+1} \times \theta_{n+1}+(1-p) \times \sum_{k=0}^{n} \frac{n+1}{n+1-k}\left(\begin{array}{l}
n \\
k
\end{array}\right) p^{k}(1-p)^{n-k} \theta_{k} .
$$

To see that $f_{n+1}\left(p_{n}\right)>0$, note that:

1. The first term on the RHS of (27) is positive,

2. The second term on the RHS of (27) is also positive in $p_{n}$. It is known that $\sum_{k=0}^{n}\left(\begin{array}{l}n \\ k\end{array}\right) p_{n}^{k}\left(1-p_{n}\right)^{n-k} \theta_{k}=0$. Observe that this sum consists of one negative term at $k=0$ followed by exclusively positive terms. The new weighing factor $(n+1) /(n+1-k)$ puts more emphasis on the latter terms since it increases as $k$ increases.

We have $f_{n+1}(0)<0$ and $f_{n+1}\left(p_{n}\right)>0$. It is known from Lemma 9 that $f_{n+1}(\cdot)$ has a unique positive real root at $p_{n+1}$. Hence $p_{n+1}$ must be in the interval $\left(0, p_{n}\right)$.

\section{Proof of Proposition 5}

The symmetric Nash equilibrium is the root of $f_{n}(\cdot)$. Remark that if $p \neq 1$ we can write

$$
f_{n}(p)=(1-p)^{n} \sum_{k=0}^{n}\left(\begin{array}{l}
n \\
k
\end{array}\right) r^{k} \theta_{k}
$$

where $r=p /(1-p)$. Note that $r$ is a monotonic transformation from $[0,1)$ to $[0, \infty)$. Since the root of $f_{n}(\cdot)$ is never equal to 1 , it is possible to focus on the roots of:

$$
g_{n}(r)=\sum_{k=0}^{n}\left(\begin{array}{l}
n \\
k
\end{array}\right) r^{k} \theta_{k}
$$

Let $r_{n}$ denote the unique positive real root of $g_{n}(\cdot)$. Note that $p_{n}=\frac{r_{n}}{1+r_{n}}$. The following Lemmas, that are needed for the proof of the Proposition, are presented in terms of $r_{n}$. 
Lemma 13 For every $n \in \mathbb{N}$, we have $r_{n+1}<\frac{n}{n+1} r_{n}$.

Proof If $g_{n+1}\left(n r_{n} /(n+1)\right)>0$, then $r_{n+1}<\frac{n}{n+1} r_{n}$. This follows from $g_{n+1}(0)<0$ and $g_{n+1}(\cdot)$ continuous. It has to be shown that $g_{n+1}\left(n r_{n} /(n+1)\right)>0$ given that $g_{n}\left(r_{n}\right)=0$. Let $r \equiv r_{n}$ and $s \equiv \frac{n}{n+1} r$ to simplify notation. Observe that:

$$
\begin{aligned}
\left(\begin{array}{l}
n \\
0
\end{array}\right) \theta_{0}+\left(\begin{array}{l}
n \\
1
\end{array}\right) r \theta_{1} & =\left(\begin{array}{c}
n \\
0
\end{array}\right) \theta_{0}+\frac{n+1}{n}\left(\begin{array}{l}
n \\
1
\end{array}\right)\left(\frac{n}{n+1} r\right) \theta_{1} \\
& =\left(\begin{array}{c}
n+1 \\
0
\end{array}\right) \theta_{0}+\left(\begin{array}{c}
n+1 \\
1
\end{array}\right) s \theta_{1}
\end{aligned}
$$

and, since $g_{n}(r)=0$ that,

$$
\left(\begin{array}{l}
n \\
0
\end{array}\right) \theta_{0}+\left(\begin{array}{l}
n \\
1
\end{array}\right) r \theta_{1}=-\left(\begin{array}{l}
n \\
2
\end{array}\right) r^{2} \theta_{2}-\cdots-\left(\begin{array}{l}
n \\
n
\end{array}\right) r^{n} \theta_{n} .
$$

Combining these two results gives:

$$
\begin{aligned}
g_{n+1}\left(\frac{n}{n+1} r\right)>0 \\
\Leftrightarrow-\left(\begin{array}{l}
n \\
2
\end{array}\right) r^{2} \theta_{2}-\cdots-\left(\begin{array}{l}
n \\
n
\end{array}\right) r^{n} \theta_{n}+\left(\begin{array}{c}
n+1 \\
2
\end{array}\right) s^{2} \theta_{2} \\
\quad+\cdots+\left(\begin{array}{c}
n+1 \\
n
\end{array}\right) s^{n} \theta_{n}+s^{n+1} \theta_{n+1}>0 \\
\Leftrightarrow-\left(\begin{array}{l}
n \\
2
\end{array}\right) r^{2} \theta_{2}-\cdots-\left(\begin{array}{l}
n \\
n
\end{array}\right) r^{n} \theta_{n}+\frac{n+1}{n-1}\left(\begin{array}{l}
n \\
2
\end{array}\right) s^{2} \theta_{2} \\
\quad+\cdots+\frac{n+1}{1}\left(\begin{array}{l}
n \\
n
\end{array}\right) s^{n} \theta_{n}+s^{n+1} \theta_{n+1}>0 \\
\Leftrightarrow \sum_{i=2}^{n}\left\{\left(\begin{array}{c}
n \\
i
\end{array}\right) \theta_{i}\left[\frac{n+1}{n+1-i} \times\left(\frac{n}{n+1}\right)^{i}-1\right] r\right\}+s^{n+1} \theta_{n+1}>0 .
\end{aligned}
$$

Since $s_{n+1} \theta_{n+1}>0$, it suffices to show that the following is true for every $n$ and for all $i=2, \ldots, n$ :

$$
\frac{n+1}{n+1-i} \times\left(\frac{n}{n+1}\right)^{i}-1 \geq 0
$$

Rearrange this to obtain:

$$
\begin{aligned}
& \frac{n^{i}}{(n+1-i)(n+1)^{i-1}}-\frac{(n+1-i)(n+1)^{i-1}}{(n+1-i)(n+1)^{i-1}} \\
& =\frac{n^{i}-(n+1-i)(n+1)^{i-1}}{(n+1-i)(n+1)^{i-1}} \geq 0
\end{aligned}
$$


which is equivalent with:

$$
n^{i}-(n+1-i)(n+1)^{i-1} \geq 0 .
$$

The rest of the proof is by induction. Let $i=2$. Now (39) boils down to $n^{2}-(n-1)$ $(n+1)=n^{2}-n^{2}+1=1 \geq 0$. Next, assume that (39) is true for an $i>2$ and show that it also is true for $i+1$. Assume that $n^{i}-(n+1-i)(n+1)^{i-1} \geq 0$ is true. It has to be shown that $n^{i+1}-(n-i)(n+1)^{i} \geq 0$ is true. Rewrite this as:

$$
n^{i+1}-(n-i)(n+1)^{i}=n\left[n^{i}-(n+1-i)(n+1)^{i-1}\right]+i(n+1)^{i-1}>0,
$$

which is true since $n^{i}-(n+1-i)(n+1)^{i-1} \geq 0$ by assumption and since $i(n+1)^{i-1}>$ 0 .

Lemma 14 If $r_{n+1}<\frac{n}{n+1} r_{n}$ and $r_{1}<1$, then for all $n$ we have $\left(1+r_{n}\right)^{n+1}>$ $\left(1+r_{n+1}\right)^{n+2}$.

Proof It is sufficient to show that this results holds for the limit case of $r_{n+1}=\frac{n}{n+1} r_{n}$. This defines a recursive relation and therefore $r_{n}$ can be rewritten as an explicit function of $n$, i.e. $r_{n}=\frac{r_{1}}{n}$. It has to be shown that:

$$
\left(1+\frac{r_{1}}{n}\right)^{n+1}>\left(1+\frac{r_{1}}{n+1}\right)^{n+2} .
$$

Let $T_{n} \equiv\left(1+\frac{r_{1}}{n}\right)^{n+1}$. Note that $\lim _{n \rightarrow \infty} T_{n}=\mathrm{e}^{r_{1}}$ since $\mathrm{e}^{x}=\lim _{n \rightarrow \infty}\left(1+\frac{x}{n}\right)^{n}$. Using the logarithmic derivative rule, it follows that:

$$
\frac{\mathrm{d} T_{n}}{\mathrm{~d} n}=\left[\log \left(1+\frac{r_{1}}{n}\right)-\frac{n+1}{n+r_{1}} \frac{r_{1}}{n}\right]\left(1+\frac{r_{1}}{n}\right)^{n+1},
$$

which has to be strictly negative. Equivalently:

$$
\log \left(1+\frac{r_{1}}{n}\right)<\frac{n+1}{n+r_{1}} \frac{r_{1}}{n}
$$

Since $r_{1} / n<1$ by assumption, we can use the following power function (Apostol 1967: pp. 388-391) to replace $\log \left(1+\frac{r_{1}}{n}\right)$ with:

$$
\frac{r_{1}}{n}+\sum_{k=1}^{\infty}\left[-\frac{1}{2 k}\left(\frac{r_{1}}{n}\right)^{2 k}+\frac{1}{2 k+1}\left(\frac{r_{1}}{n}\right)^{2 k+1}\right] .
$$

It is easily checked that $\frac{r_{1}}{n}<\frac{n+1}{n+r_{1}} \frac{r_{1}}{n}$ if $r_{1}<1$. Remark further that: 


$$
\begin{aligned}
-\frac{1}{2 k}\left(\frac{r_{1}}{n}\right)^{2 k}+\frac{1}{2 k+1}\left(\frac{r_{1}}{n}\right)^{2 k+1} & <0 \\
\Leftrightarrow-\frac{1}{2 k}+\frac{1}{2 k+1} & \times \frac{r_{1}}{n}<0 \\
\Leftrightarrow \frac{r_{1}}{n} & <\frac{2 k+1}{2 k},
\end{aligned}
$$

which is true if $r_{1}<1$. These two facts imply that

$$
\begin{aligned}
\log \left(1+\frac{r_{1}}{n}\right) & =\frac{r_{1}}{n}+\sum_{k=1}^{\infty}\left[-\frac{1}{2 k}\left(\frac{r_{1}}{n}\right)^{2 k}+\frac{1}{2 k+1}\left(\frac{r_{1}}{n}\right)^{2 k+1}\right] \\
& <\frac{r_{1}}{n}<\frac{n+1}{n+r_{1}} \frac{r_{1}}{n} .
\end{aligned}
$$

Therefore, $T_{n}$ is decreasing in $n$ if $r_{1}<1$, which concludes the proof.

Now we are able to provide the proof of Proposition 5.

Proof of part (i) We have to show that:

$$
\begin{aligned}
& \left(1-p_{n}\right)^{n+1}<\left(1-p_{n+1}\right)^{n+2} \\
\Leftrightarrow & \left(\frac{1}{1+r_{n}}\right)^{n+1}<\left(\frac{1}{1+r_{n+1}}\right)^{n+2} \\
\Leftrightarrow & \left(1+r_{n}\right)^{n+1}>\left(1+r_{n+1}\right)^{n+2} .
\end{aligned}
$$

Now if we solve (1) for $n=1$, then we see that the condition that $\left|\theta_{0}\right|<\left|\theta_{1}\right|$ is equivalent to $p_{1}<\frac{1}{2}$. Since $p_{1}<\frac{1}{2}$ implies that $r_{1}<1$ and, from Lemma 13, we know that $r_{n+1}<\frac{n}{n+1} r_{n}$, the result follows from Lemma 14 .

Proof of part (ii) Let $\hat{r}_{n} \equiv \frac{r_{1}}{n}$. Note that $r_{n}<\hat{r}_{n}$ and $\left(1+r_{n}\right)^{n+1}<\left(1+\hat{r}_{n}\right)^{n+1}$. Taking the limit we obtain:

$$
S_{\infty}=\lim _{n \rightarrow \infty}\left(1+r_{n}\right)^{n+1}<\mathrm{e}^{r_{1}}
$$

Since $\lim _{n \rightarrow \infty}\left(1+r_{n}\right)^{n+1}=\left[\lim _{n \rightarrow \infty}\left(1-p_{n}\right)^{n+1}\right]^{-1}$ and substituting $r_{1}=p_{1} /(1-$ $\left.p_{1}\right)$ leads to:

$$
\lim _{n \rightarrow \infty}\left(1-p_{n}\right)^{n+1}>\mathrm{e}^{-p_{1} /\left(1-p_{1}\right)}
$$

where the RHS is equal to $\mathrm{e}^{\theta_{0} / \theta_{1}}$.

Proof of part (iii) The expected number of participants is the probability of participation, $p_{n}$, times group size, $n+1$ (since the number of participants follows a binomial 
distribution). We have to proof that $(n+1) p_{n}<1$. Substituting $p_{n}=r_{n} /\left(1+r_{n}\right)$ yields:

$$
r_{n}<\frac{1}{n}
$$

It is known that $r_{1}<1$ and $r_{n+1}<\frac{n}{n+1} r_{n}$. Therefore $r_{n}<\frac{1}{n} r_{1}<\frac{1}{n}$.

\section{Derivation of the probability of breakdown in the counterexample}

The probability of participation is implicitly defined by:

$$
\sum_{k=0}^{n}\left(\begin{array}{l}
n \\
k
\end{array}\right) p^{k}(1-p)^{n-k} \theta_{k}=0
$$

which in this case may be rewritten as:

$$
-(1-p)^{n}+\alpha \sum_{k=1}^{n}\left(\begin{array}{l}
n \\
k
\end{array}\right)(\beta p)^{k}(1-p)^{n-k}=0
$$

If $\beta<1$, then some straightforward algebra yields:

$$
\begin{aligned}
& -(1-p)^{n}+\alpha[\beta p+(1-p)]^{n} \times \sum_{k=1}^{n}\left(\begin{array}{l}
n \\
k
\end{array}\right)\left(\frac{\beta p}{\beta p+(1-p)}\right)^{k} \\
& \times\left(\frac{1-p}{\beta p+(1-p)}\right)^{n-k}=0 .
\end{aligned}
$$

Now the part behind the summand is a proper binomial again and represents "the probability of at least one success." Exploiting this fact, we obtain:

$$
-(1-p)^{n}+\alpha[\beta p+(1-p)]^{n}\left(1-\left(\frac{1-p}{\beta p+(1-p)}\right)^{n}\right)=0,
$$

which is equivalent to:

$$
-(1-p)^{n}+\alpha[\beta p+(1-p)]^{n}-\alpha(1-p)^{n}=0
$$

which is easily solved for $p$. This yields:

$$
p_{n}=\frac{\sqrt[n]{(1+\alpha) / \alpha}-1}{\sqrt[n]{(1+\alpha) / \alpha}-(1-\beta)}
$$

Applying $S_{n}=\left(1-p_{n}\right)^{n+1}$ gives the desired result. 


\section{References}

Anderson S, Engers M (2007) Participation games: market entry, coordination, and the beautiful blonde. J Econ Behav Organ 63:120-137

Apostol T (1967) Calculus I: one-variable calculus, with an introduction to linear algebra. Wiley, New York Atkinson K (1989) An introduction to numerical analysis, 2nd edn. Wiley, New York

Dixit A, Skeath S (1999) Games of strategy. W.W Norton \& Company, New York

Haan M, Kooreman P (2003) How majorities can lose the election: another voting paradox. Soc Choice Welfare 20:509-522

Harrington J (2001) A simple game-theoretic explanation for the relationship between group size and helping. J Math Psychol 45:389-392

Kaneko M, Prokop J (1993) A game theoretical approach to the international debt overhang. J Econ 58:1-24

Mukhopadhyay K (2003) Jury size and the free rider problem. J Law Econ Organ 19:24-44

Osborne M (2004) An introduction to game theory. Oxford University Press, Oxford

Palfrey T, Rosenthal H (1983) A strategic calculus of voting. Public Choice 41:7-53

Rasmusen E (2001) Games and information. Blackwell, Malden 